$\mathbb{T}$ periodica polytechnica

Mechanical Engineering

51/2 (2007) 63-69

doi: 10.3311/pp.me.2007-2.04

web: http://www.pp.bme.hu/me

(C) Periodica Polytechnica 2007

RESEARCH ARTICLE

\section{Equivalent stress for micropolar solids}

Ákos Gombos

Received 2008-01-18

\begin{abstract}
This paper presents three definitions of equivalent stress calculation for micropolar solids. After a short review of the literature, the kinematical, equlibrium and constitutive equations are summarized. Applying the definition of the elastic strain energy, the paper gives three methods for the determination of equivalent stresses. After that, these are compared by a known cyilinder torsion analitical example from literature.
\end{abstract}

\section{Keywords}

cosserat $\cdot$ micropolar $\cdot$ equivalent stress

\section{Acknowledgement}

This research has been supported by the National Development and Research Foundation, Hungary (under Contract: OTKA, T046488). This support is gratefully acknowledged.

\section{Ákos Gombos}

Department of Applied Mechanics, BME, H-1111 Budapest, Múegyetem rkp. 5., Hungary

e-mail: gombosakos@mm.bme.hu

\section{Introduction}

Conventional formulation of continuum mechanics approaches cannot incorporate any intrinsic material length scales. However, real materials often exhibit a number of important length scales, which must be included in any realistic model: foams, granular materials, ceramics, biological tissues, fibers, particles, cellular solids and composites. Classical continuum mechanics considers the interaction of microstructural units of the material is described through stresses and displacements of material points. However interaction of grains may include rotation and associated couples-stress as well. Therefore the averaging scheme of classical continuum mechanics should consider rotation and couple stresses as well. This was the first Cosserat proposed by Eugene and Francois in their landmark publication. Their proposed theory was later reworked by Gunther and Mindlin who laid down the kinematics and statics of Cosserat continuum in a useful form for applied mechanics applications. Eringen coined the term micropolar continuum as a synonym for the Cosserat medium. Despite the obvious application to granular media, the Cosserat theory was first applied in soil mechanics applications when Muhlhaus and Vardoulakis applied the theory to localization. These authors observed that the characteristic dimension (thickness) of shear bands that are formed (in granular materials) in post localization regime is governed by the grain size. The definition of the equivalent stress is very important for the calculation of elasto-plastic theory, because the yield condition was usually based on them. In the published papers, De Borst [2], Lippmann [8], Besdo [1], Neuber [11] studied two dimensional cases. These formulas can not generalized for three dimensional. In tree dimension the equations contain more material parameters then in two dimennsional case. Forest [5] defined a general formula, but the constants what were used in the equations, are not identified by material parameters. This paper presents three different models for definitions of equivalent stress, which contain the material parameters [7], so these can be using also in numerical examples. All three definitions are based on the relation of general and uniaxial stress state, but these neglect different parts of the elastic strain energy. The yield condition used in elastoplastic theory can be determined from these 
three equivalent stress models. This paper is organized as follows. The notation for equations will be introduced at the end of this section. Section 2 describes the fundamental equations for elastic, isotropic, micropolar solids. Section 3 contains the determination of the strain energy function. In Section 4, the three equivalent stress model definitions are presented. In Section 5, a numerical example is shown for the equivalent models, using a known analitical example from literature. Regarding notation, tensors are denoted by bold-face characters, the order of which is indicated in the text. The tensor product is denoted by $\otimes$, and the following symbolic operations apply: $\mathbf{a}: \mathbf{b}=\mathrm{a}_{i j} \mathrm{~b}_{i j}$, $(\mathbf{A}: \mathbf{B})_{i j k l}=A_{i j m n} B_{m n k l}$ and $(\mathbf{C}: \mathbf{a})_{i j}=\mathrm{C}_{i j k l} \mathrm{a}_{k l}$, with the summation over repeated indices. The superscripts $T$ and -1 denote transpose and inverse, and the prefix tr refers to the trace. The symbol $\|a\|=\sqrt{\mathbf{a}^{T}: \mathbf{a}}$ is used to denote a norm of nonsymmetric second order tensor a. The second-order unit tensor (Kronecker delta) is denoted by $\boldsymbol{\delta}$ or $\delta_{i j}$, the permutation tensor $\boldsymbol{\epsilon}$ or $\epsilon_{i j k}$.

\section{Fundamental equations of linear isotropic, micropo- lar elasticity}

In this section the constitutive equations for linear micropolar elasticity are briefly reviewed. Here we use the following notations: $\mathbf{u}$ is the displacement vector, $\phi$ is the microrotation vector, $\varepsilon$ and $\gamma$ are the strain measures, $\mathbf{t}$ denote the stress tensor and $\mathbf{m}$ represent the couple stress tensor.

\subsection{Equilibrium equations}

Eringen [4] has published a comprehensive recapitulation of micropolar elasticity theory based largely on earlier papers written by him and his co-workers. His treatise will serve as the main source of the equations in this work. Only static problems will be considered. In the rectangular Cartesian tensor form, the equilibrium equations for stress $t_{k l}$ an couple stress $m_{k l}$ are written as:

$$
t_{k l, k}+f_{l}=0, \quad m_{k l, k}+\epsilon_{l m n} t_{m n}+l_{l}=0 .
$$

where $f_{l}$ and $l_{l}$ denote the body force and the body couple.

\subsection{Kinematic equations}

At any material point of the continuum, we consider both a displacement and a micro rotation vector denoted by $\mathbf{u}$ and $\phi$, respectively. The associated Cosserat deformation $\varepsilon$ and torsioncurvature tensor $\gamma$ are written by [3] p. 104., eqn (5.1.7) as:

$$
\begin{aligned}
& \boldsymbol{\varepsilon}=\operatorname{grad}^{T} \mathbf{u}+(\boldsymbol{\epsilon} \cdot \boldsymbol{\phi})^{T}, \quad\left(\varepsilon_{a b}=u_{b, a}+\epsilon_{b a c} \phi_{c}\right), \\
& \boldsymbol{\gamma}=\operatorname{grad} \boldsymbol{\phi}, \quad\left(\gamma_{a b}=\phi_{a, b}\right) .
\end{aligned}
$$

Note that $\varepsilon$ and $\boldsymbol{\gamma}$ are non-symmetric tensors.

\subsection{Constitutive relations}

The constitutive equations for linear isotropic micropolar solid and their inverse are given by

$$
\begin{aligned}
\mathbf{t} & =\lambda \operatorname{tr} \boldsymbol{\varepsilon} \boldsymbol{\delta}+(\mu+\kappa) \boldsymbol{\varepsilon}+\mu \boldsymbol{\varepsilon}^{T}, \\
\mathbf{m} & =\alpha \operatorname{tr} \boldsymbol{\gamma} \boldsymbol{\delta}+\beta \boldsymbol{\gamma}+\gamma \boldsymbol{\gamma}^{T}, \\
\boldsymbol{\varepsilon} & =-\frac{\lambda}{(2 \mu+\kappa)(3 \lambda+2 \mu+\kappa)} \operatorname{trt} \boldsymbol{\delta}+ \\
& \frac{\mu+\kappa}{\kappa(2 \mu+\kappa)} \mathbf{t}-\frac{\mu}{\kappa(2 \mu+\kappa)} \mathbf{t}^{T}, \\
\boldsymbol{\gamma} & =-\frac{\alpha}{(\beta+\gamma)(3 \alpha+\beta+\gamma)} \operatorname{trm} \boldsymbol{\delta}+ \\
& \frac{\beta}{\beta^{2}-\gamma^{2}} \mathbf{m}-\frac{\gamma}{\beta^{2}-\gamma^{2}} \mathbf{m}^{T},
\end{aligned}
$$

where $\alpha, \beta, \gamma, \mu, \kappa, \lambda$ are material parameters. These equations can be re-written in the following form:

$$
\begin{gathered}
\mathbf{t}=\mathbf{A}: \boldsymbol{\varepsilon}, \quad\left(t_{a b}=A_{a b c d} \varepsilon_{c d}\right), \\
\mathbf{m}^{T}=\mathbf{B}: \boldsymbol{\gamma}, \quad\left(m_{a b}=B_{b a c d} \gamma_{c d}\right), \\
\boldsymbol{\varepsilon}=\mathbf{A}^{-1}: \mathbf{t}, \quad\left(\varepsilon_{a b}=A_{a b c d}^{-1} t_{c d}\right), \\
\boldsymbol{\gamma}^{T}=\mathbf{B}^{-1}: \mathbf{m}, \quad\left(\gamma_{a b}=B_{b a c d}^{-1} m_{c d}\right),
\end{gathered}
$$

where the fourth order constituive tensors $A, B$ and their inverses are defined by

$$
\begin{gathered}
A_{a b c d}=\lambda \delta_{a b} \delta_{c d}+(\mu+\kappa) \delta_{a c} \delta_{b d}+\mu \delta_{a d} \delta_{b c}, \\
B_{a b c d}=\alpha \delta_{a b} \delta_{c d}+\beta \delta_{a d} \delta_{b c}+\gamma \delta_{a c} \delta_{b d}, \\
A_{a b c d}^{-1}=-\frac{\lambda}{(2 \mu+\kappa)(3 \lambda+2 \mu+\kappa)} \delta_{a b} \delta_{c d}+ \\
\frac{\mu+\kappa}{\kappa(2 \mu+\kappa)} \delta_{a c} \delta_{b d}-\frac{\mu}{\kappa(2 \mu+\kappa)} \delta_{a d} \delta_{b c}, \\
B_{a b c d}^{-1}=-\frac{\alpha}{(\beta+\gamma)(3 \alpha+\beta+\gamma)} \delta_{a b} \delta_{c d}+ \\
\frac{\beta}{\beta^{2}-\gamma^{2}} \delta_{a d} \delta_{b c}-\frac{\gamma}{\beta^{2}-\gamma^{2}} \delta_{a c} \delta_{b d} .
\end{gathered}
$$

\subsection{Material parameters}

Lakes [7] applying some experiments, defines six alternative constans from Eringen's moduli. These elastic parameters can be easily calculed from $\lambda, \mu, \kappa, \alpha, \beta, \gamma$ and vice versa by 
as

$$
\begin{gathered}
G_{m}=\frac{1}{2}(2 \mu+\kappa), \quad v_{m}=\frac{\lambda}{2 \lambda+2 \mu+\kappa}, \\
\mathcal{N}=\sqrt{\frac{\kappa}{2(\mu+\kappa)}}, \quad l_{t}=\sqrt{\frac{\beta+\gamma}{2 \mu+\kappa}}, \\
l_{b}=\sqrt{\frac{\gamma}{2(2 \mu+\kappa)}}, \quad \Psi=\frac{\beta+\gamma}{\alpha+\beta+\gamma}, \\
\alpha=\frac{2 G_{m}(1-\Psi) l_{t}^{2}}{\Psi}, \quad \beta=2 G_{m}\left(l_{t}^{2}-2 l_{b}^{2}\right), \\
\gamma=4 G_{m} l_{b}^{2}, \quad \lambda=\frac{2 G_{m} v_{m}}{1-2 v_{m}}, \\
\mu=\frac{G_{m}\left(1-2 \mathcal{N}^{2}\right)}{1-\mathcal{N}^{2}}, \quad \kappa=\frac{2 G_{m} \mathcal{N}^{2}}{1-\mathcal{N}^{2}} .
\end{gathered}
$$

Here $G_{m}$ [force/length ${ }^{2}$ ] is the Shear modulus , $v_{m}$ [dimensionless] is the Poisson ratio, $l_{t}$ and $l_{b}$ [length] are the torsion and bending Characteristic length, $\mathcal{N}$ [dimensionless] is the Coupling number and $\Psi$ [dimensionless] is the Polar ratio. The effect of these parameters are studied by Nakamura [10].

\section{Strain energy density function}

The elastic strain energy function can be defined by the product of the stress and strain and product of couple-stress and curvature-strain as:

$$
\begin{gathered}
U=\frac{1}{2} \mathbf{t}: \boldsymbol{\varepsilon}+\frac{1}{2} \mathbf{m}: \boldsymbol{\gamma}^{T}=\frac{1}{2} \mathbf{t}: \mathbf{A}^{-1}: \\
\mathbf{t}+\frac{1}{2} \mathbf{m}: \mathbf{B}^{-1}: \mathbf{m}=U_{t}(\mathbf{t})+U_{m}(\mathbf{m}),
\end{gathered}
$$

where the components $U_{t}(\mathbf{t})$ and $U_{m}(\mathbf{m})$ using the Eringen's elastic material parameters, are given by the following expressions:

$$
\begin{aligned}
U_{t}(\mathbf{t})= & \frac{1}{2}\left(-\frac{\lambda}{(2 \mu+\kappa)(3 \lambda+2 \mu+\kappa)}(\operatorname{trt})^{2}+\right. \\
& \left.\frac{\mu+\kappa}{\kappa(2 \mu+\kappa)} \mathbf{t}: \mathbf{t}-\frac{\mu}{\kappa(2 \mu+\kappa)} \mathbf{t}: \mathbf{t}^{T}\right), \\
U_{m}(\mathbf{m})= & \frac{1}{2}\left(-\frac{\alpha}{(\beta+\gamma)(3 \alpha+\beta+\gamma)}(\operatorname{trm})^{2}+\right. \\
& \left.\frac{\beta}{\beta^{2}-\gamma^{2}} \mathbf{m}: \mathbf{m}^{T}-\frac{\gamma}{\beta^{2}-\gamma^{2}} \mathbf{m}: \mathbf{m}\right) .
\end{aligned}
$$

Dividing the stress and couple-stress into deviatoric and hydrostatic parts

$$
\begin{aligned}
\mathbf{t} & =\mathbf{s}+\frac{1}{3}(\operatorname{trt}) \boldsymbol{\delta} \equiv \mathbf{s}+t_{h} \boldsymbol{\delta} \\
\mathbf{m} & =\mathbf{m}_{d}+\frac{1}{3}(\operatorname{trm}) \boldsymbol{\delta} \equiv \mathbf{m}_{d}+m_{h} \boldsymbol{\delta}
\end{aligned}
$$

then, the strain energy can be expressed in the following form:

$$
U(\mathbf{t}, \mathbf{m})=U_{t d}(\mathbf{s})+U_{m d}\left(\mathbf{m}_{d}\right)+U_{t h}\left(t_{h}\right)+U_{m h}\left(m_{h}\right),
$$

where the deviatoric $\left(U_{t d}(\mathbf{s})\right.$ and $\left.U_{m d}\left(\mathbf{m}_{d}\right)\right)$ and hidrostatic $\left(U_{t h}\left(t_{h}\right)\right.$ and $\left.U_{m h}\left(m_{h}\right)\right)$ parts of strain energy can be defined

$$
\begin{aligned}
U_{t d}(\mathbf{s}) & =\frac{1}{2}\left(\frac{\mu+\kappa}{\kappa(2 \mu+\kappa)} \mathbf{s}: \mathbf{s}-\frac{\mu}{\kappa(2 \mu+\kappa)} \mathbf{s}: \mathbf{s}^{T}\right), \\
U_{m d}\left(\mathbf{m}_{d}\right) & =\frac{1}{2}\left(\frac{\beta}{\beta^{2}-\gamma^{2}} \mathbf{m}_{d}: \mathbf{m}_{d}^{T}-\frac{\gamma}{\beta^{2}-\gamma^{2}} \mathbf{m}_{d}: \mathbf{m}_{d}\right), \\
U_{t h}\left(t_{h}\right) & =\frac{3}{2}\left(\frac{1}{\kappa+3 \lambda+2 \mu} t_{h}^{2}\right), \\
U_{m h}\left(m_{h}\right) & =\frac{3}{2}\left(\frac{1}{3 \alpha+\beta+\gamma} m_{h}^{2}\right) .
\end{aligned}
$$

Using the symmetric and anti-symmetric parts of the stress and couple-stress, the formulas (22) and (23) can be written as follows

$$
\begin{aligned}
U_{t d}(\mathbf{s}) & =\frac{1}{2}\left(\frac{1}{2 \mu+\kappa} \mathbf{s}_{S}: \mathbf{s}_{S}+\frac{1}{\kappa} \mathbf{s}_{A}: \mathbf{s}_{A}\right) \\
U_{m d}\left(\mathbf{m}_{d}\right) & =\frac{1}{2}\left(\frac{1}{\beta+\gamma} \mathbf{m}_{d S}: \mathbf{m}_{d S}-\frac{1}{\beta-\gamma} \mathbf{m}_{d A}: \mathbf{m}_{d A}\right) .
\end{aligned}
$$

Using the material parameters defined in (2.4), Eringen [4] gives the nessesary and sufficient conditions that the strain energy $U$ be nonnegative as follows

$$
\begin{array}{lll}
0 \leq 3 \lambda+2 \mu+\kappa, & 0 \leq 2 \mu+\kappa, & 0 \leq \kappa, \\
0 \leq 3 \alpha+2 \beta+\gamma, & 0 \leq \beta+\gamma, & 0 \leq \gamma,
\end{array}
$$

which can also be defined with the Lakes parameters as

$$
\begin{array}{r}
0 \leq \Psi \leq \frac{3}{2}, \quad l_{t} \geq 0, \quad l_{b} \geq 0, \quad 2 l_{b} \geq l_{t} \\
0 \leq \mathcal{N} \leq 1, \quad-1 \leq v_{m} \leq \frac{1}{2}
\end{array}
$$

\section{The equivalent stress definitions}

The formula (21) is the elastic strain energy function in a general, three dimensional case. For uniaxial stress state

$$
\tilde{t}_{a b}=\sigma \delta_{1 a} \delta_{1 b}, \quad \widetilde{s}_{a b}=\sigma\left(\delta_{1 a} \delta_{1 b}-\frac{1}{3} \delta_{a b}\right)
$$

the strain energy can be exepressed by 


$$
U^{1 D}=U_{t d}^{1 D}+U_{t h}^{1 D}=\frac{1}{3(2 \mu+\kappa)} \sigma^{2}+\frac{1}{6(\kappa+3 \lambda+2 \mu)} \sigma^{2} .
$$

The equivalent stress definition are based on the comparison of uniaxial and the three dimensional cases.

\section{Model 1.}

This model supposes that the full strain energy is equivalent in uniaxial one, namely (31) and (21) are equals,

$$
\left.\begin{array}{l}
U^{1 D}(\sigma)=U_{t d}^{1 D}(\sigma)+U_{t h}^{1 D}(\sigma) \\
U(\mathbf{t}, \mathbf{m})=U_{t d}(\mathbf{s})+U_{m d}\left(\mathbf{m}_{d}\right)+U_{t h}\left(t_{h}\right)+U_{m h}\left(m_{h}\right)
\end{array}\right\} \quad U^{1 D}(\sigma)=U(\mathbf{t}, \mathbf{m}) .
$$

From (32) it follows that

$$
\sigma=\sqrt{\frac{6(\kappa+3 \lambda+2 \mu)(2 \mu+\kappa)}{2(\kappa+3 \lambda+2 \mu)+\xi(2 \mu+\kappa)}\left[U_{t d}(\mathbf{s})+U_{m d}\left(\mathbf{m}_{d}\right)+U_{t h}\left(t_{h}\right)+U_{m h}\left(m_{h}\right)\right]} .
$$

Substitute Eqs. (22) - (25) and (31) into (33), the final form of the equivalent stress is

$$
\bar{\sigma}^{(1)}=\sqrt{\frac{3}{2} a_{1}\left[\mathbf{s}_{S}: \mathbf{s}_{S}+a_{2} \mathbf{s}_{A}: \mathbf{s}_{A}+a_{3} \mathbf{m}_{d S}: \mathbf{m}_{d S}+a_{4} \mathbf{m}_{d A}: \mathbf{m}_{d A}+a_{5} m_{h}^{2}+a_{6} t_{h}^{2}\right]}
$$

where the constants are defined by

$$
\begin{aligned}
& a_{1}=\frac{2(\kappa+3 \lambda+2 \mu)}{2(\kappa+3 \lambda+2 \mu)+(2 \mu+\kappa)} \equiv \frac{2\left(1+v_{m}\right)}{3}, \quad a_{2}=\frac{2 \mu+\kappa}{\kappa} \equiv \frac{1-\mathcal{N}^{2}}{\mathcal{N}^{2}}, \\
& a_{3}=\frac{2 \mu+\kappa}{\beta+\gamma} \equiv \frac{1}{l_{t}^{2}}, \quad a_{4}=-\frac{2 \mu+\kappa}{\beta-\gamma} \equiv \frac{1}{4 l_{b}^{2}-l_{t}^{2}}, \\
& a_{5}=\frac{3(2 \mu+\kappa)}{3 \alpha+\beta+\gamma} \equiv \frac{3 \Psi}{(3-2 \Psi) l_{t}^{2}}, \quad a_{6}=\frac{3(2 \mu+\kappa)}{\kappa+3 \lambda+2 \mu} \equiv \frac{9}{1+v_{m}}-6 .
\end{aligned}
$$

\section{Model 2.}

This model supposes that some parts of strain energy is not important for equivalent stress. The hydrostatical part from uniaxial case and the part of the general case related to stress, are not included. This means

$$
\left.\begin{array}{l}
U^{1 D}(\sigma)=U_{t d}^{1 D}(\sigma) \\
U(\mathbf{s}, \mathbf{m})=U_{t d}(\mathbf{s})+U_{m d}\left(\mathbf{m}_{d}\right)+U_{m h}\left(m_{h}\right)
\end{array}\right\} \quad U^{1 D}(\sigma)=U(\mathbf{s}, \mathbf{m}) .
$$

From (38) it follows that

$$
\sigma=\sqrt{3(2 \mu+\kappa)\left[U_{t d}(\mathbf{s})+U_{m d}\left(\mathbf{m}_{d}\right)+U_{m h}\left(m_{h}\right)\right]} .
$$

Substituting Eqs. (22), (23), (25) and(31) into(38), the final form for equivalent stress is

$$
\bar{\sigma}^{(2)}=\sqrt{\frac{3}{2}\left(\mathbf{s}_{S}: \mathbf{s}_{S}+a_{2} \mathbf{s}_{A}: \mathbf{s}_{A}+a_{3} \mathbf{m}_{d S}: \mathbf{m}_{d S}+a_{4} \mathbf{m}_{d A}: \mathbf{m}_{d A}+a_{5} m_{h}^{2}\right)},
$$

where constant $a_{2}, a_{3}, a_{4}$ and $a_{5}$ are the same as in the first model (35) - (37). 
Model 3.

This model contains more suppositions than the second one. It neglects the hidrostatical part from uniaxial case again and hidrostaticial part of stress and couple stress part of general case. This means that (31) without $U_{t h}^{1 D}$ is equal to (21) without $U_{t h}$ and $U_{m h}$.

$$
\left.\begin{array}{l}
U^{1 D}(\sigma)=U_{t d}^{1 D}(\sigma) \\
U\left(\mathbf{s}, \mathbf{m}_{d}\right)=U_{t d}(\mathbf{s})+U_{m d}\left(\mathbf{m}_{d}\right)
\end{array}\right\} \quad U^{1 D}(\sigma)=U\left(\mathbf{s}, \mathbf{m}_{d}\right)
$$

From (41) it follows that

$$
\sigma=\sqrt{3(2 \mu+\kappa)\left(U_{t d}(\mathbf{s})+U_{m d}\left(\mathbf{m}_{d}\right)\right)}
$$

Combining Eqs (22), (23) and(31) into(42), the finally form for equivalent stress is

$$
\bar{\sigma}^{(3)}=\sqrt{\frac{3}{2}\left(\mathbf{s}_{S}: \mathbf{s}_{S}+a_{2} \mathbf{s}_{A}: \mathbf{s}_{A}+a_{3} \mathbf{m}_{d S}: \mathbf{m}_{d S}+a_{4} \mathbf{m}_{d A}: \mathbf{m}_{d A}\right)}
$$

where constants $a_{2}, a_{3}$ and $a_{4}$ are defined by (35), (36). Note that Forest [5] presented a similar formula for equivalent stress, but he has applied the total couple-stress instead of its deviatoric part.

\section{Numerical example. Torsion and tension of solid circular cylinder}

The boundary-value problems deal with cylinders of radius $R$ and length $c$ subjected to either axial tension $P$ and torsion $T$. The analytical solution in cylindrical coordinates $(r, \Theta, z)$ is given by Gauthier and Jashman [6]. The boundary conditions are: $r=R$, $P=\int_{A} t_{z z} d A, \mathrm{~T}=\int_{A}\left(r t_{z \Theta}+m_{z z}\right) d A$ and $z=0$. The solution is given by

$$
\begin{aligned}
& t_{z z}=\frac{P}{A} \\
& t_{\theta z}=(2 \mu+\kappa) \frac{r C_{1}}{2}-\kappa C_{9} I_{1}(p r) \\
& t_{z \theta}=(2 \mu+\kappa) \frac{r C_{1}}{2}+\kappa C_{9} I_{1}(p r) \\
& m_{r r}=(\alpha+\beta+\gamma) p C_{9} I_{0}(p r)-\frac{\beta+\gamma}{r}\left(C_{9} I_{1}(p r)+\frac{r C_{1}}{2}\right), \\
& m_{\theta \theta}=\alpha p C_{9} I_{0}(p r)+\frac{\beta+\gamma}{r}\left(C_{9} I_{1}(p r)-\frac{r C_{1}}{2}\right), \\
& m_{z z}=\alpha p C_{9} I_{0}(p r)+(\beta+\gamma) C_{1}, \\
& C_{1}=2 C_{9}\left(\frac{\alpha+\beta+\gamma}{\beta+\gamma} p I_{0}(p R)-\frac{1}{R} I_{1}(p R)\right) \text {, } \\
& C_{9}=\frac{T}{2 A\left((\alpha+\beta+\gamma)\left(\frac{3}{2}+k\right) p I_{0}(p R)-(2+k)(\beta+\gamma) \frac{I_{1}(p R)}{R}\right)}, \\
& p^{2}=\frac{2 \kappa}{\alpha+\beta+\gamma}, \quad k=\frac{R^{2}(\kappa+2 \mu)}{4(\beta+\gamma)},
\end{aligned}
$$


where $I_{n}()$ is the modified Bessel function of the first kind of order $\mathrm{n}$.

For this problem, using (44) - (52) and (34), (40), (43) the equivalent stresses can be expressed by

$$
\begin{aligned}
\bar{\sigma}^{(1)} & =\left\{\frac { 2 ( 1 + v _ { m } ) } { 3 } \left(\frac{\left(m_{r r}-m_{\theta \theta}\right)^{2}+\left(m_{r r}-m_{z z}\right)^{2}+\left(m_{z z}-m_{\theta \theta}\right)^{2}}{2 l_{t}^{2}}+\frac{3\left(t_{z \theta}-t_{\theta z}\right)^{2}}{4 \mathcal{N}^{2}}\right.\right. \\
& \left.\left.+3 t_{z \theta} t_{\theta z}+\frac{\Psi\left(m_{r r}+m_{\theta \theta}+m_{z z}\right)^{2}}{2(2-\Psi) l_{t}^{2}}+\frac{3 t_{z z}^{2}}{2\left(1+v_{m}\right)}\right)\right\}^{\frac{1}{2}}, \\
\bar{\sigma}^{(2)} & =\left\{\frac{\left(m_{r r}-m_{\theta \theta}\right)^{2}+\left(m_{r r}-m_{z z}\right)^{2}+\left(m_{z z}-m_{\theta \theta}\right)^{2}}{2 l_{t}^{2}}+\frac{\Psi\left(m_{r r}+m_{\theta \theta}+m_{z z}\right)^{2}}{2(2-\Psi) l_{t}^{2}}\right. \\
& \left.+\frac{3\left(t_{z \theta}-t_{\theta z}\right)^{2}}{4 \mathcal{N}^{2}}+3 t_{z \theta} t_{\theta z}+t_{z z}^{2}\right\}^{\frac{1}{2}}, \\
\bar{\sigma}^{(3)} & =\left\{\frac{\left(m_{r r}-m_{\theta \theta}\right)^{2}+\left(m_{r r}-m_{z z}\right)^{2}+\left(m_{z z}-m_{\theta \theta}\right)^{2}}{2 l_{t}^{2}}+\frac{3\left(t_{z \theta}-t_{\theta z}\right)^{2}}{4 \mathcal{N}^{2}}+3 t_{z \theta} t_{\theta z}+t_{z z}^{2}\right\}^{\frac{1}{2}},
\end{aligned},
$$

where $\bar{\sigma}^{1}, \bar{\sigma}^{2}$ and $\bar{\sigma}^{3}$ correspond to the models $1-3$, respectively. Using the material parameters $G_{m}=1033[M P a], v_{m}=0.34$, $l_{t}=0.65[\mathrm{~mm}], l_{b}=0.4[\mathrm{~mm}], N=0.6, \Psi=1.5$, the distribution of equivalent stress on radius are presented by the following figure.

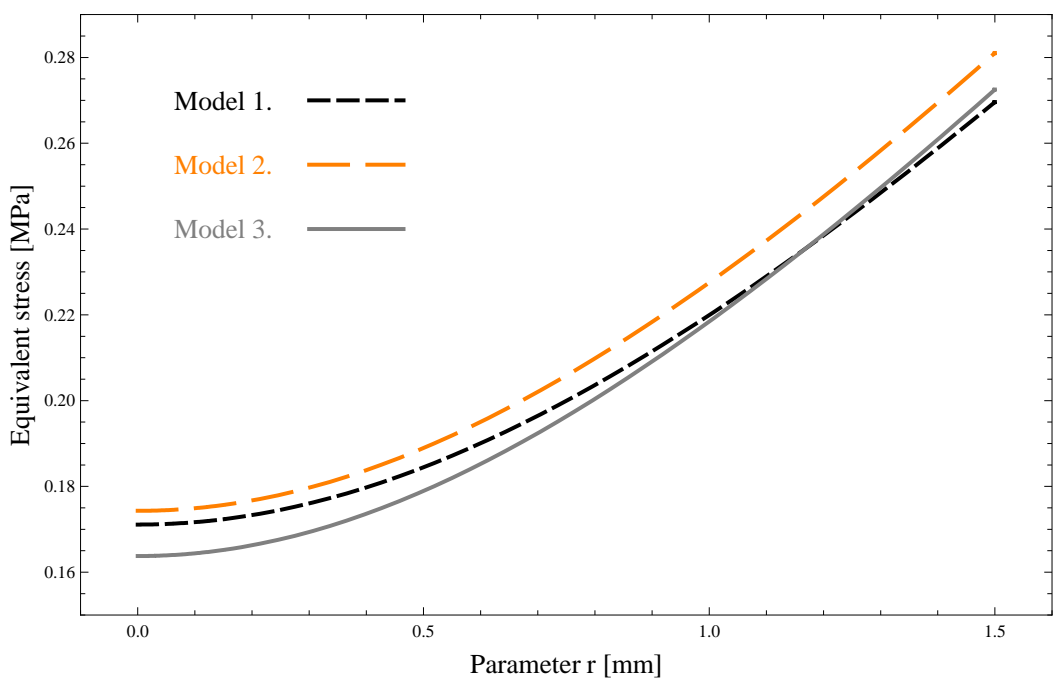

Fig. 1. Variation of equivalent stresses vs. radius of the cylinder according to Models 1-3

Fig. 1 shows that for the torsion problem, there are no significant differences between the equivalent stress models which are introduced in this paper. 


\section{Conclusion}

A brief summary of the fundamental equations of elastic, micropolar solids is given in this paper. It defines the elastic strain energy which is based on the additive decomposition of stress and couple stress parts. Using this definition, the paper introduces three different models for equivalent stress, which are based on the comparison of uniaxial and general stress state. Furthermore numerical differences have been shown between the three models applying a known analitical solution (torsion of a circular cylinder). The comparison shows that there are no significant differences with the used material parameters for this problem. This formulas can be applicable to yield condition for the elastoplastic micropolar model.

\section{References}

1 Besdo D., Ein Beitrag zur nichtlinearen Theorie des Cosserat-Kontinuums, Acta Mech. 20 (1974), 105-131.

2 De Borst R, A generalisation of $J_{2}$-flow theory for polar continua, Comp. Meth. Appl. Mech. Eng. 103 (1993), 347-362.

3 Eringen A C, Microcontinuum Field Theories, Foundations and Solids, Springer Verlag, Berlin, 1999.

4 , Theory of Micropolar Elasticity, Fracture (Liebowitz H, ed.), Vol. 2, Academic Press, New York, 1968, pp. 621-729.

5 Forest S, Cosserat Media, Encyclopedia of Materials:Science and Technology, Elsevier, 2001, pp. 1715-1718.

6 Gauthier R D, Jashman W E, A Quest for Micropolar Elastic Constans, ASME J. Appl. Mech. 43 (1975), 369-374.

7 Lakes R S, Experimental methods for study of Cosserat elastic solids and other generalized elastic continua, Continuum Models for Materials with Microstructure., 1995, pp. 1-25.

8 Lippmann H, Cosserat plasticity and plastic spin, ASME Appl. Mech. Rev. 48 (1995), 753-762.

9 , Eine Cosserat-Theorie des plastischen Fließens, Acta Mech. 8 (1969), 255-284.

10 Nakamura S, Lakes R S, Finite element analysis of Saint Venant end effect in micropolar elastic solids, Engineering Computations 12 (1995), 571-587.

11 Neuber H, Über Probleme der Spannungskonzentration im CosseratKörper, Acta Mechanica 2 (1966), 48-69. 